\title{
Contemporary pharmacogenetic assays in view of the PharmGKB database
}

\author{
Laurentijn Tilleman ${ }^{1}$, Jana Weymaere ${ }^{1}$, Björn Heindryckx², Dieter Deforce ${ }^{\ddagger 1}$ \& Filip Van \\ Nieuwerburgh*, $\ddagger, 1$ \\ ${ }^{1}$ Laboratory of Pharmaceutical Biotechnology, Ghent University, Ottergemsesteenweg 460, 9000 Ghent, Belgium \\ ${ }^{2}$ Ghent-Fertility \& Stem Cell Team (G-FaST), Department for Reproductive Medicine, Ghent University Hospital, Corneel \\ Heymanslaan 10, 9000 Ghent, Belgium \\ *Author for correspondence: filip.vannieuwerburgh@ugent.be \\ ${ }^{\ddagger}$ Authors contributed equally
}

\begin{abstract}
Aim: Six modern PGx assays were compared with the Pharmacogenomics Knowledge Base (PharmGKB) to determine the proportion of the currently known PGx genotypes that are assessed by these assays. Materials \& methods: Investigated assays were 'Ion AmpliSeq Pharmacogenomics,' 'iPLEX PGx Pro,' 'DMET Plus', 'PharmcoScan', 'Living DNA' and '23andMe.' Results: PharmGKB contains 3474 clinical annotations of which 75, 70 and $45 \%$ can be determined by PharmacoScan, Living DNA and 23andMe, respectively. The other assays are designed to test a specific subset of PGx variants. Conclusion: Assaying all known PGx variants would only comprise a minor fraction of the current assays' capacity. Unfortunately, this is not achieved. Moreover, not necessarily the variants with the highest effects or the highest evidence are selected.
\end{abstract}

First draft submitted: 16 October 2018; Accepted for publication: 26 November 2018; Published online: 18 March 2019

Keywords: $\quad$ ADME $\bullet$ pharmacogenetics $\bullet$ pharmacogenomics $\bullet$ PharmGKB

In recent years, hundreds of genetic association studies have explored the relationship between common genetic variation and drug response. The results of these studies were collected in different pharmaco-genetic/-genomic (PGx) databases, for instance, the Pharmacogenomics Knowledge Base (PharmGKB) database, SNPedia and the PharmacoGenomic Mutation Database (PGMD) [1-3]. The PharmGKB database is an initiative to gather all currently reported variant-drug interactions in a public knowledge base. Each variant-drug interaction is curated by at least two different scientific reviewers. The PharmGKB database can be accessed via the Web interface or by the application programming interface (API) $[1,4,5]$. SNPedia is a Wiki-based database of human genetic variants. The content on the Wiki pages is cited with peer-reviewed scientific publications. Curation is done by Wiki users, including the editors, and two independently developed software bots. It is used by Promethease [2] to create a personal report, which links the genetic variants, determined by for example direct-to-consumer (DTC) genetics services, to the information that is published about them. The SNPedia Wiki data can be accessed via the Web interface or by the API $[2,6,7]$. The PGMD is a comprehensive manually curated pharmacogenomics database specialized to link sequencing data to PGx data. The data of the PGMD are not freely accessible [3]. PharmGKB is to our knowledge the currently most comprehensive public PGx database, with the best annotated variant-drug interactions. It has also a freely accessible and easy-to-use API. We used this knowledge base as a reference to check how many of the currently known PGx variant-drug interactions can be determined using the current commercially available PGx assays.

\section{PharmGKB}

The PharmGKB database is a curated database of PGx relationships between genetic variations and drug-response. The PharmGKB curators review articles from the past and current scientific literature. First, each article is curated individually to extract the variant-drug interactions, which leads to variant annotations. Later, variant annotations from different studies are combined into a PharmGKB clinical annotation (CA) with a certain level of evidence. 


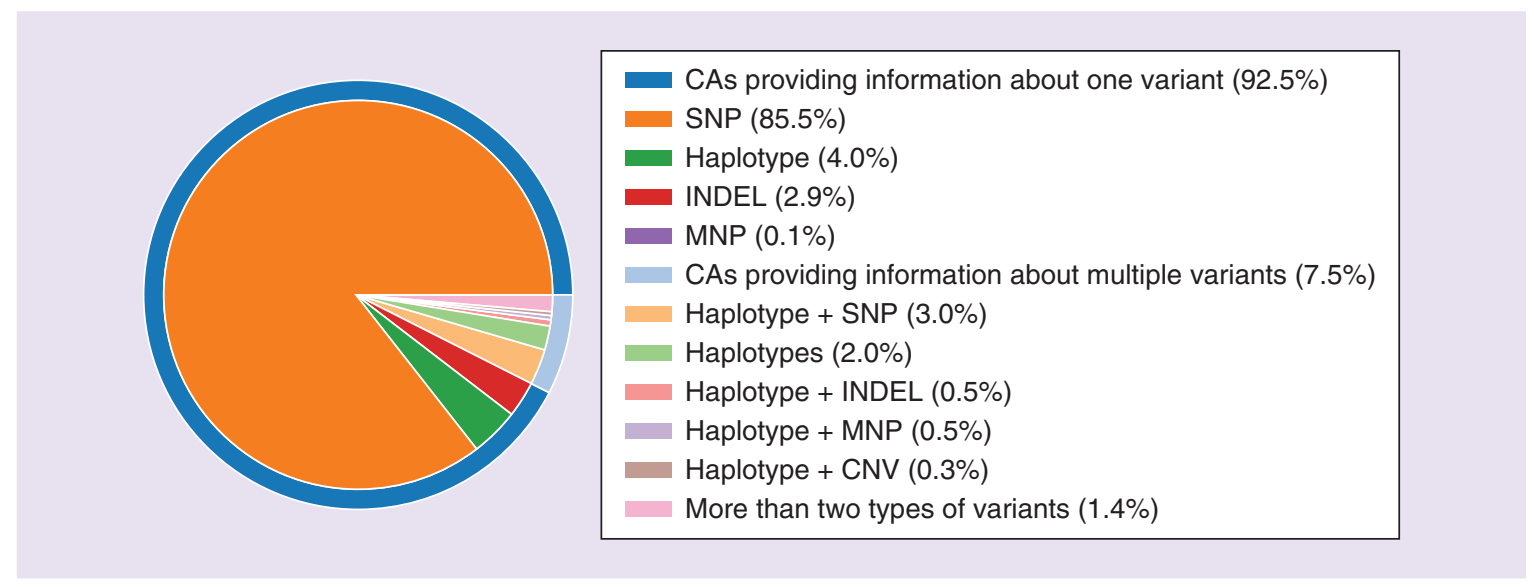

Figure 1. Clinical annotations classified according to the type of genetic variant that is described. CA: Clinical annotation; CNV: Copy number variations; INDEL: Insertions and deletions; MNP: Multiple nucleotide polymorphisms.

For color figures please see online at: www.futuremedicine.com/doi/10.2217/pgs-2018-0167

There are six evidence levels: $1 \mathrm{~A}, 1 \mathrm{~B}, 2 \mathrm{~A}, 2 \mathrm{~B}, 3$ and 4 . Level 4 is the lowest. This level contains the CAs that are based on a case report, a nonsignificant study, or a study with only in vitro evidence. Level 3 contains annotations for a variant-drug combination based on a single significant study or multiple studies that lack clear evidence of an association. Level 2B contains annotations for a variant-drug combination with moderate evidence of an association. The associations must be replicated but the effect size may be small, and/or some studies do not show statistical significance. In level $2 \mathrm{~A}$, the same criteria are applied as in level $2 \mathrm{~B}$, but the associated variant is within a very important pharmacogene (VIP). Level $1 \mathrm{~B}$ contains the CAs with evidence that is replicated in more than one study, with a high significance level and a strong effect size. Level 1A contains annotations for a variant-drug combination in a Clinical Pharmacogenetic Implementation Consortium (CPIC) [8] or medical society-endorsed PGx guideline or implemented at a Pharmacogenomics Research Network [9] site or in another major health system. In the PharmGKB database, some genes are selected as VIPs after extensive reviewing of a variety of sources, such as the US FDA biomarker list, FDA-approved drug labels with pharmacogenetic information and genes which were included in CPIC guidelines. These genes have either a role in the metabolism of many drugs or contain variants that potentially contribute to a severe drug response. For each VIP, a summary is made. These summaries are a succinct pharmacogenetic-based overview of the literature, which covers background information about the gene structure, physiological role of the encoded protein, disease associations and provide a collective view on pharmacokinetics and pharmacodynamics. These summaries are published in PGx journals $[1,4,10]$.

The PharmGKB database currently contains 19,028 variant annotations, which resulted in 3474 CAs in six evidence levels: 53, 16, 116, 119, 2795 and 375 CAs in evidence level 1A, 1B, 2A, 2B, 3 and 4, respectively. Those CAs describe the interaction between 932 genes and 567 drugs. In total, there are 2086 SNPs, 264 haplotypes, 55 insertions and deletions (INDELs), eight multiple nucleotide polymorphisms (MNPs) and six copy number variations $(\mathrm{CNVs})$ for which there is a CA. Figure 1 shows a pie chart illustrating the proportions of CAs that provide PGx information about these different genetic variant types. CAs can provide information about one variant or about multiple variants. Most of the CAs provide information about SNPs (89.2\%). This is not surprising as most of the past PGx literature is based on techniques that genotype individual SNPs. Examples of prevalent techniques in the PGx literature are single-base extension SNP genotyping, TaqMan SNP genotyping assays and SNP microarrays [11-13]. While these techniques can be used to genotype a large set of SNPs, they normally do not allow haplotyping as it remains unknown if individual SNPs occur together on a single allele. With the advent of Massively Parallel Sequencing (MPS), haplotyping, INDEL, alternative splicing and genome-wide CNV analyses became more feasible. Long-read MPS offers even more possibilities, allowing phasing of entire genes. The PGx literature is lagging behind these technological developments. While MPS became available more than a decade ago, its routine application to analyze genes beyond individual SNP genotyping occurred more recently [14]. 
Figure 2. Venn diagram of number of genes in the core absorption, distribution, metabolism and excretion list (Core ADME), genes in the Extended ADME list, VIP and genes with a clinical annotation (PharmGKB). ADME: Absorption, distribution, metabolism and excretion; VIP: Very important pharmacogenes.

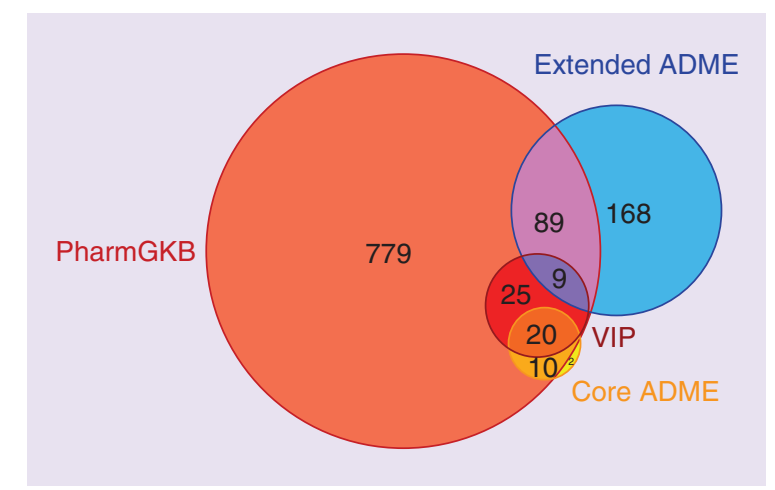

PharmaADME core \& extended absorption, distribution, metabolism \& excretion list

A multidisciplinary group of representatives from the pharmaceutical industry and an academic center, the PharmaADME consortium, developed a consensus list of important PGx genes that cause common drug-gene interactions involved in drug absorption, distribution, metabolism and excretion (ADME) [15]. This consortium compiled a Core ADME list of 32 genes and an Extended ADME list of 266 genes. Genes that were added to the Core ADME list must fit all of the following criteria: genes need to be directly involved in the drug metabolism and/or need to influence a drug's pharmacokinetic profile, genes need to be identified by the FDA as a validated biomarker, the effect of the gene variants on the gene function needs to be supported by multiple scientific publications from different research groups, the fact that gene variants result in an altered protein function is supported by Key Opinion Leaders and genes must not be involved in disease predisposition or prognosis. Genes that were added to the Extended ADME list do not meet one of the first four criteria. These lists can be found in the PharmaADME database [15-17]. When the genes of the Core and Extended ADME list are compared with the genes with a CA, it is remarkable that there is not a CA for every gene in the Core or Extended ADME list. Furthermore, not all the VIPs in the PharmGKB database are member of one of the two ADME lists. Figure 2 shows a Venn diagram of number of genes in the Core ADME list, genes in the Extended ADME list, VIPs and genes with a CA.

\section{Current PGx genotyping methods}

Different methods can be applied to analyze a patient's PGx profile. Whole-genome sequencing can be performed to detect genome-wide SNPs, haplotypes, INDELs, MNPs and CNVs. While this method provides the most comprehensive data, the cost of this approach is currently still prohibitive. A more cost-efficient method is targeted sequencing. DNA from genomic regions of interest such as the exome or a specific set of PGx genes can be enriched from the extracted DNA. This is routinely performed using a pool of hybridizing oligonucleotides or by multiplex PCR [18-20]. These methods are still cost-prohibitive to be routinely applied. Currently, many PGx assays are based on microarray technology. These allow analysis of hundreds to hundreds of thousands of individual predefined positions in the genome. Arrays are designed to include small genomic variants of interest, based on for example a PGx database such as PharmGKB. The advantage of these methods is the low cost when applied in large-scale facilities, however only small genetic variants, such as SNPs, are detected. As more than $90 \%$ of currently known PGx variants are SNPs, this disadvantage is somewhat mitigated. The design of the assay used is important. As our understanding of the genome function grows exponentially, microarray designs need to be constantly updated.

In this study, six contemporary, commercially available assays were compared with the PharmGKB database. Ion AmpliSeq Pharmacogenomics (Thermo Fisher Scientific, MA, USA) uses a single pool of primers to perform a multiplex PCR of specific 70 to 190 bp regions around 138 well-documented SNPs, INDELs and CNVs of 40 PGx genes [21] and is part of a next-generation sequencing pipeline. iPLEX PGx Pro uses the MassARRAY (Agena Bioscience, CA, USA) to detect SNPs, INDELs and CNVs. Samples are analyzed in eight multiplex wells, each with predesigned PCR primers, extension primers and iPLEX Pro reagents. Haplotype reports are automatically generated using MassARRAY analysis software. This assay detects 192 variants of 36 PGx genes [22]. DMET Plus (Thermo Fisher Scientific) is a molecular inversion probe-based PGx microarray and covers most of the PharmaADME Core list. This assay detects 1936 SNPs, CNVs and INDELs of 231 genes [23,24]. The PharmacoScan Solution (Thermo Fisher Scientific) is a microarray that scans for a broad spectrum of genomic variations. This assay detects most of the variants of the CPIC guidelines, the PharmGKB database, the PharmaADME Core list 
Table 1. Comparison of the six pharmacogenetic assays.

\begin{tabular}{|c|c|c|c|c|c|c|}
\hline Assays & 23andMe & Living DNA & PharmacoScan & DMET Plus & $\begin{array}{l}\text { Ion AmpliSeq } \\
\text { Pharmacogenomics }\end{array}$ & iPLEX PGx Pro \\
\hline iPLEX PGx Pro & 51 & 111 & 171 & 168 & 67 & $192^{\dagger}$ \\
\hline $\begin{array}{l}\text { Ion AmpliSeq } \\
\text { Pharmacogenomics }\end{array}$ & 44 & 108 & 132 & 102 & $138^{\dagger}$ & \\
\hline DMET Plus & 702 & 537 & 1871 & $1919^{\dagger}$ & & \\
\hline PharmacoScan & 28,334 & 21,715 & $115,483^{\dagger}$ & & & \\
\hline LivingDNA & 171,183 & $632,214^{\dagger}$ & & & & \\
\hline 23andMe & $713,599^{\dagger}$ & & & & & \\
\hline
\end{tabular}

and other additional variants [25]. Living DNA and 23andMe are DTC genotyping assays. Living DNA uses a customized Illumina Infinium Global Screening Array assaying 632,214 SNPs. 23andMe uses the Illumina Human OmniExpress BeadChip array, which detects 713,599 SNPs. These DTC assays provide genotyping data that are associated with a broad range of health, nutrition, disease, ancestry and PGx traits [26,27]. These six assays are designed to genotype individual SNPs, MNPs, INDELs and CNVs. Different software packages were developed to combine these SNPs, MNPs, INDELs and CNVs to haplotypes. Because these variants are not phased, it is not possible to unambiguously determine the correct haplotype. Therefore, only variants that are individual SNPs, MNPs, INDELs or CNVs are considered in the evaluation of the assays. Because many haplotypes composed of more than one SNP, MNP, INDEL or CNV are important pharmacogenetic markers, we discuss the impact of PGx information that is restricted to individual variants in the Discussion section.

\section{Materials \& methods}

A local version of the PharmGKB database was built to facilitate the analysis. The data were downloaded via the API [5]. The Core and Extended ADME list were downloaded from the PharmaADME web page [15] and added to the local version of the PharmGKB database. CAs were categorized in different groups: CAs described in a VIP, CAs described in a gene of the Core ADME list, CAs described in a gene of the Extended ADME list and CAs categorized by their level of evidence.

The list of the dbSNP reference identifiers (rsids) [28] from the variants, which were tested in each PGx assay, was downloaded from different sources. Ion AmpliSeq Pharmacogenomics variants list was downloaded from the vendor's website [29]. The list of variants from the iPLEX PGx Pro Panel was obtained from the Supplementary Materials of Jensen et al. [30]. For the DMET Plus, the variant list release 32 was downloaded from the Thermo Fisher website [31]. Release 35 of the annotation file for the SNPs of the PharmacoScan was downloaded from the Thermo Fischer website [32]. 23andMe uses the Illumina Infinium Human OmniExpress BeadChip array. A list with the SNPs was downloaded from the Illumina website [33]. Living DNA provided a list of their custom SNPs of their Illumina Infinium Global Screening Array. The rsids were used to compare the assays with each other and the PharmGKB database. Only variants that are individual SNPs, MNPs, INDELs or CNVs, were considered in the comparisons.

\section{Results}

Number of analyzed genetic variants

The total number of genetic variants analyzed by each assay was compared. Table 1 shows the total number of genetic variants analyzed by each assay. The number of variants analyzed in common between two assays is also shown. Supplementary Table 1 shows the percentages of analyzed variants that are analyzed in common with the other assays.

The DTC assays, 23andMe and Living DNA, provide genotyping data associated with health, nutrition, disease, ancestry and PGx traits and analyze a number of variants that are 5- to 5000-times higher than the other studied assays that are specifically designed to analyze PGx variants. It is remarkable that, although Living DNA and 23andMe both use BeadChip arrays that are designed to capture as many known variants as possible, only about $25 \%$ of the variants analyzed by each assay are analyzed in common. This is explained in part by the different BeadChip array design strategies used by both assays: 23andMe uses the Infinium HumanOmniExpress BeadChip 
array, which is optimized to contain SNP content from all three HapMap phases that have been selected to capture the greatest amount of common variation and drive the discovery of novel associations with traits and diseases [34]. Living DNA uses the Infinium Global Screening Array of which the content was selected for high imputation accuracy at minor allele frequencies of more than $1 \%$ across all 261000 Genomes Project populations. The clinical research content includes variants with established disease associations, relevant pharmacogenomics markers and curated exonic content based on ClinVar, NHGRI, PharmGKB and ExAC databases [35]. Nevertheless, there does not seem to be a consensus on which genetic variants are most informative in DTC testing. Furthermore, it is remarkable that only a small percentage of the variants that are targeted by the other PGx assays are included in the DTC assays. As both BeadChip array designs have a custom marker add-on capacity of 30-50 thousand, it seems that the DTC assays do not use their full potential regarding PGx variant analysis.

The PGx assays that are commercialized by Thermo Fisher Scientific (Ion AmpliSeq Pharmacogenomics, DMET Plus and PharmacoScan) are specifically designed to target PGx variants. By design, the number of analyzed variants differs substantially between these assays. PharmacoScan analyses more than a hundred thousand variants, while DMET Plus and Ion AmpliSeq Pharmacogenomics analyze 1919 and 138 variants, respectively. Almost all variants analyzed by Ion AmpliSeq Pharmacogenomics and DMET Plus are also analyzed by PharmacoScan. Ion AmpliSeq Pharmacogenomics and DMET Plus also overlap to a high extent.

The iPLEX PGx assays are also designed to target PGx variants. The targeted variants show a lot of overlap with PharmacoScan, DMET Plus and Living DNA.

\section{Number of analyzed genetic variants that are described in a PharmGKB CA}

The PGx assays were compared with the PharmGKB database based on the genetic variants that are described in a PharmGKB CA. Variants were categorized in different groups: variants that are described in a CA with a specific level of evidence, variants in a VIP, variants in a Core ADME gene and variants in an Extended ADME gene. An overview of the number of variants per category that is tested by each assay is given in Table 2 .

Although the DTC assays are not specifically designed to analyze PGx variants, Living DNA analyzes the highest number of PGx variants, $64.9 \%$ of the variants described in a PharmGKB CA. 23andMe analyzes substantially less, genotyping only $36.7 \%$ of the variants described in a CA. As discussed in the previous section, this can be attributed to the fact that Living DNA is using a BeadChip design that is more tailored to include variants described in databases such as PharmGKB. As both BeadChip array designs can add 30-50 thousand extra variants, it is surprising that not more $\mathrm{PGx}$ variants are included. Adding all PharmGKB's PGx variants to the BeadChip design would entail less than $1 \%$ of a BeadChip's capacity.

PharmacoScan is a microarray-based PGx tool targeting more than hundred thousand variants. Because it is specifically designed to target PGx variants, it is surprising that it only includes 1554 (64.2\%) of the 2419 variants described by a CA. The usefulness of the more than hundred thousand other analyzed variants is not clear. On par with Living DNA, PharmacoScan provides the most comprehensive data on variants with a CA. The other investigated PGx assays genotype less than $50 \%$ of these variants. These assays are designed to analyze a specific subset of PGx variants. It is not clear how this selection was made. Table 2 shows that the variants with the highest effects or the highest evidence are not necessarily selected.

\section{Number of determined PharmGKB CAs}

In the previous paragraph and in Table 2, the assays were evaluated based on their ability to detect variants with a CA. In this paragraph, the assays are in an analogous way compared based on the number of CAs that can be determined by the different assays. The number of variants with a CA and the number of CAs are not necessarily identical. One genetic variant can influence more than one drug and thus have multiple CAs. Furthermore, one CA can include more than one genetic variant.

Table 3 describes the number of CAs that can be determined by each assay. In this table, CAs are classified by their level of evidence and divided in CAs belonging to a VIP, a Core ADME gene and an Extended ADME gene. By comparing Tables 2 and 3, the difference between number of assayed PGx variants and the number of CAs that can be determined is illustrated. While Living DNA assayed the most PGx variants, PharmacoScan can determine most CAs. In addition, Ion AmpliSeq Pharmacogenomics, DMET Plus and iPLEX PGx Pro can determine proportionally more CAs than it assays PGx variants. This indicates that the PGx variants determined by these dedicated PGx tools provide information on a high number of CAs and are thus relatively important PGx variants. 


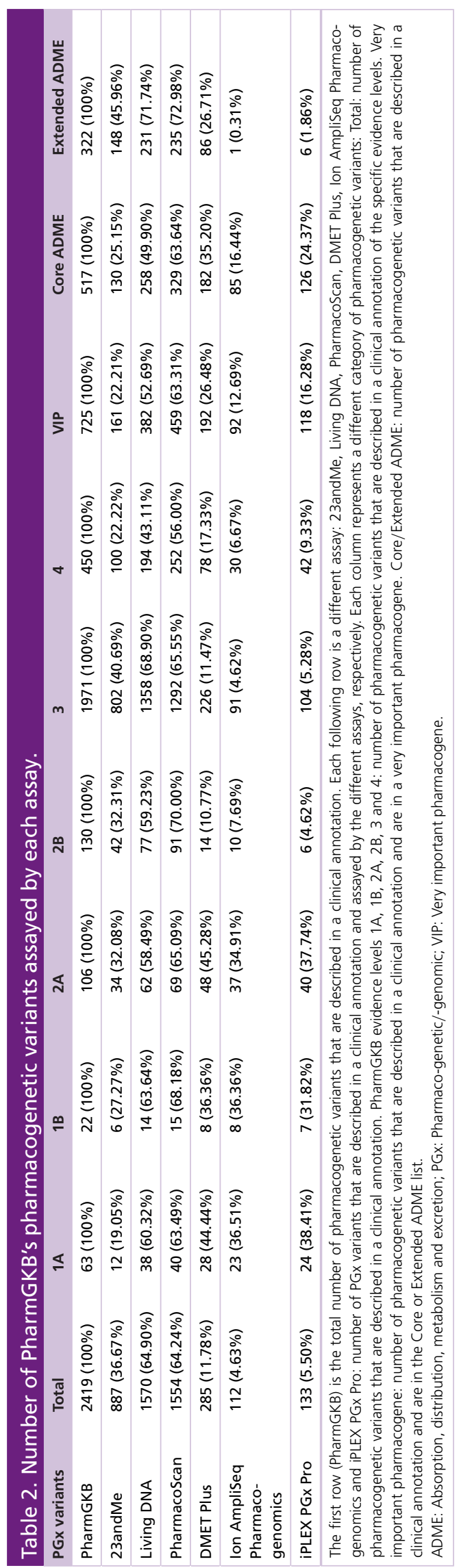




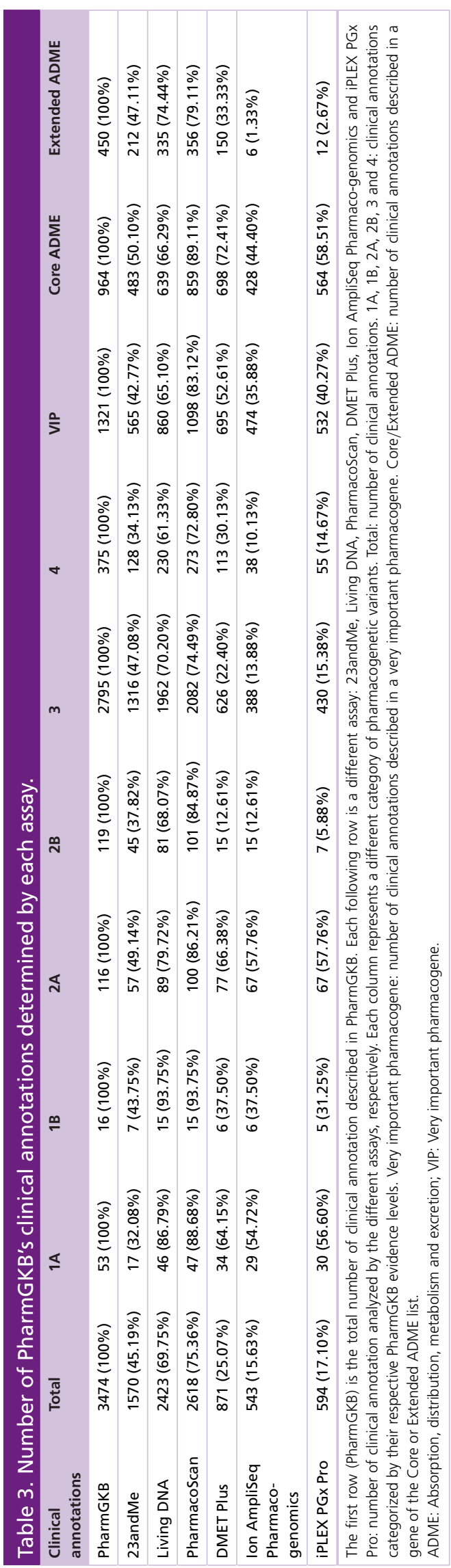


Table 4. Number of drugs covered by each assay.

\begin{tabular}{|c|c|c|c|c|c|c|c|}
\hline Evidence level & PharmGKB & 23andMe & Living DNA & PharmacoScan & DMET Plus & $\begin{array}{l}\text { Ion AmpliSeq } \\
\text { Pharmaco- } \\
\text { genomics }\end{array}$ & iPLEX PGx Pro \\
\hline All evidence levels & $567(100 \%)$ & $105(18.52 \%)$ & $189(33.33 \%)$ & $247(43.56 \%)$ & $53(9.35 \%)$ & $32(5.64 \%)$ & $34(6.00 \%)$ \\
\hline $\begin{array}{l}\text { Evidence levels } 1 A \text {, } \\
1 B, 2 A \text { and } 2 B\end{array}$ & $179(100 \%)$ & $38(21.23 \%)$ & $91(50.84 \%)$ & $121(67.60 \%)$ & $29(16.20 \%)$ & $19(10.61 \%)$ & $18(10.06 \%)$ \\
\hline
\end{tabular}

\section{Drugs of which all PGx variants are analyzed}

In a clinical context, it is important to have the complete picture of the PGx variants that affect a specific drug since drugs can have multiple relevant variant-drug interactions. Therefore, it is valuable to know which assays provide complete coverage for which drugs. A drug is considered as completely covered at all evidence levels if all PGx variants can be determined by the assay. Similarly, a drug is considered as completely covered at evidence levels 1A, 1B, 2A and 2B if all PGx variants with that evidence level can be determined by the assay. Table 4 gives an overview of the number of drugs with a PharmGKB CA that are completely covered by each assay at different evidence levels. A list with the corresponding drug names is given in Supplementary Table 2.

PharmacoScan, which determines the highest number of the CAs $(75.36 \%$ of all CAs), covers only $43.56 \%$ of the drugs with a CA completely. Most of the missing CAs have a relatively low evidence level of 3 or 4 . Of the 567 drugs in PharmGKB, 179 are described in CAs with an evidence level of 1A, 1B, 2A or 2B. PharmacoScan covers $67.60 \%$ of the drugs at this evidence level. Similar results are obtained for the other studied assays.

\section{Discussion}

Our study puts the data that are provided by current contemporary, commercially available PGx assays in the perspective of current PGx knowledge. We show that these assays determine only a small part of currently known clinical annotations. Furthermore, we show that even the most extensive tests, such as PharmacoScan, do not provide the complete PGx picture for many drugs. One of the reasons for this is the fact that the techniques used by the studied assays can only detect individual unphased SNPs, MNPs, INDELs and CNVs and are therefore not capable to unambiguously detect all haplotypes that are defined by a combination of variants in the same allele. Haplotypes can nonetheless be imputed from unphased individual variants [36]. There are several software packages available to impute genotypes from genotyping arrays to reference panels. Each tool provides specific pros and cons, in terms of speed and accuracy. Also, the considered set of reference alleles has a large impact on the imputation results. While imputation is a commonly used technique in, for example, genome-wide association studies (GWAS), the fact remains that the haplotypes are not directly observed. The imputed haplotypes are a statistical 'best guess' and are prone to errors. For example in GWAS studies with thousands of samples, these errors are a manageable problem. When performing haplotype imputation on an individual, with the intention to make informed decisions for that individual, these errors are a problem. Efforts to validate pharmacogenetic methods that include imputation have been published [37]. Specifically, haplotypes that have one or a few defining variants can be correctly imputed. Comparison of the assays, including their haplotype imputation capabilities, raises questions that are not the scope of this study. Some of the assays like PharmacoScan genotype, many of the variants that are part of a haplotype and come with a proprietary haplotype imputation method. It could have been an option to include imputed results in this study. Results of the imputation methods are, however, highly dependent on the chosen reference alleles in that method. When considering a small set of reference alleles, it can be possible to unambiguously impute one of those alleles from the provided unphased variant data. However, when a larger, more detailed set of reference alleles is considered that same data can sometimes not unambiguously impute the reference alleles. Hence, results cannot be easily compared between methods provided by different assays [38]. Validating the precision and accuracy of the proprietary methods is also a possible issue, which is out of the scope of this study. Another option would be to impute haplotypes using the same open-source software [39] in combination with one reference allele set for all methods. Developing, implementing and testing such a method would be a study in itself.

Haplotypes that are defined by a combination of variants in the same allele, are necessary to determine the phenotype of several extremely important drug-metabolizing enzymes and transporters such as ABCB1, CYP2A6, 
CYP2C19, G6PD, GSTM1, SLCO1B1 and TPMT and comprise 10.9\% of the variants in PharmGKB, which are described in $11.1 \%$ of the CAs. Notwithstanding this low number of haplotypes with a CA, they have a high impact on the predictive value of the PGx data: $35.4 \%$ of the drugs are described in a CA comprising a haplotype. One of the most important examples is CYP2D6, which metabolizes approximately 20-25\% of the drugs present in the liver and $11 \%$ of the drugs with a CA in PharmGKB [40,41]. The CYP2D6 gene is highly polymorphic with over 100 star $\left(^{*}\right)$ alleles in the PharmVar database [42]. Due to the high number of alleles and the complexity of the different alleles, haploblocks are needed to precisely genotype the CYP2D6 gene. In addition, the CYP2D6 gene has two highly homologous neighboring pseudogenes, CYP2D7 and CYP2D8, which makes it difficult to distinguish variants from these three genes [43].

For clinical implementation of pharmacogenetic data, it is not only important to have a complete picture of all the genetic variants that affect a drug but it is equally important to know which pathways are involved to what extent. Not all gene-drug interactions are equally important. For example, some drugs are metabolized by several enzymes whereby each enzyme only partially metabolizes the drug. An example is ondansetron, which is metabolized for 70\% by CYP1A2, CYP2D6 and CYP3A to 6-hydroxyondanstron, 7-hydroxyondansetron and 8-hydroxyondansetron, whereby each enzyme can catalyze the reaction from ondansetron to each metabolite [44]. A variant in the gene of one of these enzymes will only partially affect the drug's metabolization, even when that variant has a severe impact on the enzyme. In this example, the variants of CYP2D 6 are described in a CA with a level of evidence $1 \mathrm{~A}$ while the variants of $C Y P 3 A$ are described in a CA with a level of evidence 3 . As described above, a variant-drug interaction with a high level of evidence has enough scientific power to assume that these variants affect the drug metabolism, but this does not mean that the effect on the drug metabolism is high. In the metabolism of ondansetron, CYP3A is the main metabolizing enzyme. Variants in CYP3A might have more impact on ondansetron metabolization than variants in CYP2D6, which only metabolizes around $20 \%$ of ondansetron present in the liver $[44,45]$. Although there is higher scientific evidence for the variants in CYP2D6 to have a PGx effect on the drug's metabolization, variants in $C Y P 3 A$ might be more relevant to the patient in this case.

To some extent, the studied assays seem to incorporate variants with relatively high alternative allele frequencies, neglecting variants with low alternative allele frequencies (data not shown). Approximately $84 \%$ of the variants in PharmGKB have a known allele frequency. More than $50 \%$ of the variants in PharmGKB have an allele frequency lower than $25 \%$ for the alternative allele, and $90 \%$ of the variants described in CAs with evidence level $1 \mathrm{~A}$ have an allele frequency lower than $20 \%$ for the alternative allele [28]. The number of affected people by these variants is not large, but the effect of these variants on their drug treatment can be of high importance. Therefore, the alternative allele frequency should not be a criterion whether to include a variant in an assay or not.

There are many techniques available to genotype pharmacogenes. Most commercial tests, such as the assays discussed in this study, are genotyping individual SNPs, MNPs, INDELs and CNVs. A disadvantage of this type of assays is that they cannot unambiguously determine haplotypes that are necessary to unravel the genotypes of important pharmacogenes. At this moment, no commercial PGx kits are available that provide the necessary haplotypes. Therefore, new techniques could be used and developed, such as long-read sequencing and haploblock sequencing. Oxford Nanopore Technologies (Oxford, UK) and PacBio (Pacific Biosciences, CA, USA) provide long-read sequencing with reads of up to $800 \mathrm{~kb}$ long. 10X Genomics (Pleasanton, CA, USA) can also phase variants into large haploblocks of up to $10 \mathrm{~kb}$ [46-48]. A drawback of sequencing techniques might be the introduction of sequence errors. These can be introduced when performing targeted PCR, during library preparation or during sequencing. Fortunately, targeted PCR can be performed using high-fidelity polymerases. Alternatively, hybridization-based target enrichment can be performed. Oxford nanopore and PacBio sequencing allow PCRfree library preparation, but unfortunately, they have relatively high sequencing error rates. This can, in part, be compensated by sequencing with enough coverage [49]. 10X Genomics technology can phase variants into large haploblocks of up to $10 \mathrm{~kb}$ with the quality of Illumina short reads sequencing, but unfortunately, this involves an elaborate sample preparation workflow including hybridization-based target enrichment, clonal amplification in gel-beads, library preparation with PCR-enrichment and Illumina sequencing.

\section{Conclusion}

In this study, six commercially available PGx or DTC assays were compared with the PGx variants and the CAs described in the PharmGKB database, the core ADME list and the extended ADME list. Although the DTC assays are not specifically designed to analyze PGx variants, Living DNA genotypes the highest percentage (64.9\%) of PGx variants described by a CA in PharmGKB. 23andme genotypes substantially less (36.7\%) of these variants. As 
the BeadChip array designs that are used by Living DNA and 23andMe can add 30-50 thousand extra variants, it is surprising that not more PGx variants are included. Adding all of PharmGKB's PGx variants to the BeadChip design would entail less than $1 \%$ of a BeadChip's capacity. PharmacoScan is a microarray-based PGx tool targeting more than hundred thousand variants and is specifically designed to target PGx variants. Remarkably, it only includes 1554 (64.2\%) of the 2419 variants described by a CA. The other investigated PGx assays are designed to genotype a specific subset of PGx variants. It is not clear how this selection was made. Our analysis shows that it is not necessarily the variants with the highest effects or the highest evidence that are selected. Of clinical importance is the fact that the PGx assays do not provide results for all PGx variants of most drugs, even not for the PGx variants with high evidence levels.

\section{Summary points}

\section{Pharmaco-genetic/-genomic literature reference}

- We used the Pharmacogenomics Knowledge Base (PharmGKB) as a reference to check how many of the currently known pharmaco-genetic/-genomic (PGx) variant-drug interactions can be determined using the current commercially available PGx and genome-wide direct-to-consumer (DTC) assays.

- The PharmGKB database currently contains 19,028 variant annotations, which resulted in 3474 clinical annotations (CAs) in six evidence levels: 53, 16, 116, 119, 2795 and 375 CAs in evidence level 1A, 1B, 2A, 2B, 3 and 4 , respectively. Those CAs describe the interaction between 932 genes and 567 drugs. In total, there are 2086 SNPs, 264 haplotypes, 55 insertions and deletions, eight multiple nucleotide polymorphisms and six copy number variations for which there is a CA.

Current PGx genotyping methods

- In this study, six contemporary, commercially available assays were compared with the PharmGKB database: Ion AmpliSeq Pharmacogenomics, a next-generation sequencing assay; iPlex PGx Pro, a mass spectrometry-based assay; DMET Plus, a Molecular Inversion Probe-based microarray; PharmacoScan Solution, a microarray; Living DNA and 23andMe, DTC microarrays.

Results

- Although DTC assays are not specifically designed to analyze PGx variants, Living DNA genotypes the highest number of PGx variants, genotyping $64.90 \%$ of the variants described in a CA.

- In a clinical context, it is important to have the complete picture of the PGx variants that affect a specific drug since drugs can have multiple relevant variant-drug interactions. PharmacoScan, which determines the highest number of the CAs ( $75.36 \%$ of all CAs), covers only $43.56 \%$ of the drugs with a CA completely. Most of the missing CAs have a relatively low evidence level of 3 or 4 . Of the 567 drugs in PharmGKB, 179 are described in CAs with an evidence level of 1A, 1B, 2A, or 2B. PharmacoScan covers $67.60 \%$ of the drugs at this evidence level.

- DTC and specifically designed PGx assays like Pharmacoscan target up to hundred thousand PGx variants. Remarkably, it only includes $1554(64.2 \%)$ of the 2419 variants described by a CA. Not necessarily the variants with the highest effects or the highest evidence are examined by the assays.

Financial \& competing interests disclosure

The authors have no relevant affiliations or financial involvement with any organization or entity with a financial interest in or financial conflict with the subject matter or materials discussed in the manuscript. This includes employment, consultancies, honoraria, stock ownership or options, expert testimony, grants or patents received or pending or royalties.

No writing assistance was utilized in the production of this manuscript.

\section{References}

1 McDonagh EM, Whirl-Carrillo M, Garten Y, Altman RB, Klein TE. From pharmacogenomic knowledge acquisition to clinical applications: the PharmGKB as a clinical pharmacogenomic biomarker resource. Biomark. Med. 5(6), 795-806 (2011).

2 Cariaso M, Lennon G. SNPedia: a wiki supporting personal genome annotation, interpretation and analysis. Nucleic Acids Res. 40(D1), D1308-D1312 (2012).

3 Kaplun A, Hogan JD, Schacherer F et al. PGMD: a comprehensive manually curated pharmacogenomic database. Pharmacogenomics J. 16(2), 124-128 (2016).

4 The Pharmacogenomics Knowledge Base. www.pharmgkb.org

5 PharmGKB API. https://api.pharmgkb.org/

6 SNPedia. https://snpedia.com/

7 SNPedia API. https://bots.snpedia.com/api.php 
Relling MV, Klein TE. CPIC: Clinical Pharmacogenetics Implementation Consortium of the Pharmacogenomics Research Network. Clin. Pharmacol. Ther. 89(3), 464-467 (2011).

9 Relling M, Krauss R, Roden D et al. New Pharmacogenomics Research Network: an open community catalyzing research and translation in precision medicine. Clin. Pharmacol. Ther. 102(6), 897-902 (2017).

10 Whirl-Carrillo M, McDonagh EM, Hebert JM et al. Pharmacogenomics knowledge for personalized medicine. Clin. Pharmacol. Ther. 92(4), 414-417 (2012).

11 Nikiforov TT, Rendle RB, Goelet P et al. Genetic Bit analysis: a solid phase method for typing single nucleotide polymorphisms. Nucleic Acids Res. 22(20), 4167-4175 (1994).

12 McGuigan FEA, Ralston SH. Single nucleotide polymorphism detection:allelic discrimination using TaqMan. Psychiatr. Genet. 12(3), 133-136 (2002).

13 LaFramboise T. Single nucleotide polymorphism arrays: a decade of biological, computational and technological advances. Nucleic Acids Res. 37(13), 4181-4193 (2009).

14 Ji Y, Si Y, McMillin GA, Lyon E. Clinical pharmacogenomics testing in the era of next generation sequencing: challenges and opportunities for precision medicine. Expert Rev. Mol. Diagn. 18(5), 411-421 (2018).

16 Sim SC, Altman RB, Ingelman-Sundberg M. Databases in the area of pharmacogenetics. Hum. Mutat. 32(5), 526-531 (2011).

17 Koshy B. Gaining Consensus on emerging science: the ADME panel as an exemplar. 24 (2008). www.ema.europa.eu/docs/en_GB/document_library/Presentation/2009/11/WC500010651.pdf

18 Comino-Méndez I, Gracia-Aznárez FJ, Schiavi F et al. Exome sequencing identifies MAX mutations as a cause of hereditary pheochromocytoma. Nat. Genet. 43(7), 663-667 (2011).

19 Mertes F, ElSharawy A, Sauer $S$ et al. Targeted enrichment of genomic DNA regions for next-generation sequencing. Brief Funct. Genomics 10(6), 374-386 (2011).

20 Zheng Z, Liebers M, Zhelyazkova B et al. Anchored multiplex PCR for targeted next-generation sequencing. Nat. Med. 20(12), 1479-1484 (2014).

21 Complete next-generation sequencing solution for pharmacogenomics research: introducing Ion AmpliSeq Pharmacogenomics Research Panel (2015). https://tools.thermofisher.com/content/sfs/brochures/Ion-AmpliSeq-PGx-Research-Panel-Flyer.pdf

22 Everts RE, Metzler H, Huang V, Honisch C, Nunez R. Development and research validation of the iPLEX ${ }^{\circledR}$ ADME PGx panel on the MassARRAY ${ }^{\circledR}$ system. Biotech. Protoc. Guid. 2-6 (2012).

23 Arbitrio M, Di Martino MT, Scionti F et al. DMET $^{\text {TM }}$ (Drug Metabolism Enzymes and Transporters): a pharmacogenomic platform for precision medicine. Oncotarget 7(33), 54028-54050 (2016).

24 Burmester JK, Sedova M, Shapero MH, Mansfield E. DMET ${ }^{\mathrm{TM}}$ microarray technology for pharmacogenomics-based personalized medicine. In: Methods in Molecular Biology, Volume 632. Chittur SV (Ed.). Humana Press, Totowa, NJ, USA, 99-124 (2010).

25 PharmacoScan Solution Preemptive genotyping of known pharmacogenomics markers in a single assay (2017). https://tools.thermofisher.com/content/sfs/manuals/PG1673-PJT2289-COL13268-PharmacoScan-Data-Sheet.pdf Living DNA. www.livingdna.com/

27 23andMe. www.23andme.com

28 Sherry ST. dbSNP: the NCBI database of genetic variation. Nucleic Acids Res. 29(1), 308-311 (2001).

$29 \mathrm{PgGx}$ AmpliSeq Panel Content. https://tools.thermofisher.com/content/sfs/brochures/PgGx-AmpliSeq-Panel-Content.xlsx Jensen L, Børsting C, Dalhoff K, Morling N. Evaluation of the iPLEX ${ }^{\circledR}$ ADME PGx Pro Panel and allele frequencies of pharmacogenetic markers in Danes. Clin. Biochem. 49(16-17), 1299-1301 (2016).

31 DMET Plus release 32 annotations. https://sec-assets.thermof isher.com/TFS-Assets/LSG/Support-Files/DMET_Plus-na32-annot-csv.zip

32 PharmacoScan release 35 annotations. https://sec-assets.thermof isher.com/TFS-Assets/LSG/Support-Files/PharmacoScan-na35-annot-csv.zip

33 Infinium Omniexpress-24 v1.2 Manifest File. ftp://webdata2:webdata2@ussd-ftp.illumina.com/downloads/productfiles/humanomniex press-24/v1-2/infinium-omniexpress-24-v1-2-manifest-file-csv.zip

34 Infinium OmniExpress-24 v1.2 BeadChip Commercial Data Sheet (2016). www.illumina.com/documents/products/datasheets/datasheet_human_omni_express.pdf

35 Infinium ${ }^{\circledR}$ Global Screening Array-24 v1.0 Commercial Data Sheet (2017). www.illumina.com/content/dam/illumina-marketing/docu ments/products/datasheets/infinium-commercial-gsa-data-sheet-370-2016-016.pdf

36 Das S, Abecasis GR, Browning BL. Genotype imputation from large reference panels. Ann. Rev. Genomics Hum. Genet. 19(1), 73-96 (2018). 
37 Pratt VM, Everts RE, Aggarwal P et al. Characterization of 137 genomic DNA reference materials for 28 pharmacogenetic genes. J. Mol. Diagnostics 18(1), 109-123 (2016).

38 Samwald M, Blagec K, Hofer S, Freimuth RR. Analyzing the potential for incorrect haplotype calls with different pharmacogenomic assays in different populations: a simulation based on 1000 Genomes data. Pharmacogenomics 16(15), 1713-1721 (2015).

39 Browning BL, Zhou Y, Browning SR. A one-penny imputed genome from next-generation reference panels. Am. J. Hum. Genet. 103(3), 338-348 (2018).

40 Zhou S-F, Liu J-P, Lai X-S. Substrate specificity, inhibitors and regulation of human cytochrome P450 2D6 and implications in drug development. Curr. Med. Chem. 16(21), 2661-2805 (2009).

41 Chen Q, Wei D. Human cytochrome P450 and personalized medicine. In: Advance in Structural Bioinformatics. Wei D, Xu Q, Zhao T, Dai H (Eds). Springer, Dordrecht, The Netherlands, 827 (2015).

42 Gaedigk A, Ingelman-Sundberg M, Miller NA, Leeder JS, Whirl-Carrillo M, Klein TE. The Pharmacogene Variation (PharmVar) Consortium: incorporation of the human cytochrome P450 (CYP) allele nomenclature database. Clin. Pharmacol. Ther. 103(3), 399-401 (2018).

43 Kimura S, Umeno M, Skoda RC, Meyer UA, Gonzalez FJ. The human debrisoquine 4-hydroxylase (CYP2D) locus: sequence and identification of the polymorphic CYP2D6 gene, a related gene, and a pseudogene. Am. J. Hum. Genet. 45(6), 889-904 (1989).

44 Dixon CM, Colthup PV, Serabjit-Singh CJ et al. Multiple forms of cytochrome P450 are involved in the metabolism of ondansetron in humans. Drug Metab. Dispos. 23(11), 1225-1230 (1995).

45 Lewis DFV, Ito Y, Eddershaw PJ, Dickins M, Goldfarb PS. An evaluation of ondansetron binding interactions with human cytochrome P450 enzymes CYP3A4 and CYP2D6. Drug Metab. Lett. 4(1), 25-30 (2010).

46 Yang Y, Botton MR, Scott ER, Scott SA. Sequencing the CYP2D6 gene: from variant allele discovery to clinical pharmacogenetic testing. Pharmacogenomics 18(7), 673-685 (2017).

47 Jain M, Koren S, Miga KH et al. Nanopore sequencing and assembly of a human genome with ultra-long reads. Nat. Biotechnol. 36(4), 338-345 (2018).

48 Porubsky D, Garg S, Sanders AD et al. Dense and accurate whole-chromosome haplotyping of individual genomes. Nat. Commun. 8(1), 1293 (2017).

49 Beretta S, Patterson MD, Zaccaria S, Della Vedova G, Bonizzoni P. HapCHAT: adaptive haplotype assembly for efficiently leveraging high coverage in long reads. BMC Bioinformatics 19(1), 252 (2018). 